\title{
Lithium abundances in halo dwarfs
}

\author{
Sean G. Ryan ${ }^{1} \dagger$ \\ ${ }^{1}$ Department of Physics \& Astronomy, Open University, Walton Hall MK7 6AA, UK \\ email: s.g.ryan@open.ac.uk
}

\begin{abstract}
The pioneering observations of Spite \& Spite showed lithium abundances in halo dwarfs to be almost uniform, irrespective of metallicity and mass over a range of effective temperatures from $\sim 5600 \mathrm{~K}$ up to the main-sequence turnoff. They inferred that the observed abundance was "hardly altered" from that produced in the hot Big Bang. Subsequent efforts have endeavoured to determine how small or large "hardly" could be. Simplistic arguments based on the uniformity of the Spite plateau suggest there should only be a small difference between the Big Bang lithium abundance and the observationally inferred plateau value, whereas more physical lines of reasoning suggest the difference could be more substantial. This review paper discusses observational and theoretical developments.
\end{abstract}

Keywords. Nuclear reactions, nucleosynthesis, abundances; stars: abundances; stars: Population II; stars: subdwarfs; Galaxy: abundances; Galaxy: halo; cosmology: early universe

\section{Introduction}

Ever since Spite \& Spite (1982) announced their discovery that halo dwarfs warmer than $\sim 5600 \mathrm{~K}$ had essentially uniform lithium abundances, hardly altered from the value inherited by the stars from the hot Big Bang, astronomers have questioned just how close the observationally inferred abundance is to the primordial one (Boesgaard \& Steigman 1985). Over the subsequent twenty-plus years, this issue has not been adequately resolved. Certainly there has been progress on observational and theoretical fronts, but it is not clear which, if any, of the proposed interpretations is the correct one to explain the $\mathrm{Li}$ abundances of what have become known as the Spite plateau stars. Perhaps the situation is similar to that which pertained to the uncertain value of the Hubble constant prior to the completion of the key programme on the HST: the value of the Hubble constant was known reasonably accurately, but at that time we didn't know by whom.

There have been several recent reviews of lithium in halo dwarfs (Lambert 2004, Ryan \& Elliott 2005), so this report will concentrate on the most recent developments where possible. I shall for the most part ignore ${ }^{6} \mathrm{Li}$ which is discussed in several others papers in this volume.

\section{Spectroscopy as a probe of stellar structure and evolution}

Stellar spectra, from which Li abundance measurements are made, directly probe only the thin stellar photosphere. The photosphere is representative of the whole surface convection zone to the extent that mixing timescales in this zone, including the photosphere, are short compared to the lithium destruction timescales of main-sequence stars. It is clear that the photosphere is unrepresentative of the layers deeper than the surface convection zone where temperatures greatly exceed $2.6 \times 10^{6} \mathrm{~K}$, at which temperature ${ }^{7} \mathrm{Li}$

$\dagger$ Address from October 2005: School of Physics, Astronomy \& Mathematics, U. Hertfordshire, College Lane, Hatfield AL10 9AB, UK 
is destroyed in ${ }^{7} \mathrm{Li}(\mathrm{p}, \alpha)^{4} \mathrm{He}$ reactions. A star's photospheric $\mathrm{Li}$ abundance will decrease if $\mathrm{Li}$ is destroyed in or removed from the surface convection zone. The former happens when the surface convective zone is deep enough to reach regions at $2.6 \times 10^{6} \mathrm{~K}$. This condition is almost certainly fulfilled during a star's pre-main-sequence phase, though the amount of Li destruction that actually occurs depends on uncertain stellar structure and evolution issues. For stars that are now halo dwarfs, calculations of the amount of pre-main-sequence and main-sequence destruction of lithium have ranged from very little $(\simeq$ few $\times 0.01$ dex; Deliyannis, Demarque \& Kawaler 1990) to quite a lot $(\simeq$ few $\times 0.1$ dex; Pinsonneault, Deliyannis \& Demarque 1992) depending on the mechanisms by which material is mixed and their effectiveness. In contrast, removal of material from the surface convective zone could, and perhaps even should, be effected by diffusion. Under this mechanism Li preferentially sinks, by virtue of its atomic weight, from the surface convection zone to deeper layers where it will survive if the temperature does not exceed the $2.6 \times 10^{6} \mathrm{~K}$ limit (Deliyannis \& Demarque 1991, Salaris \& Weiss 2001). The surface convection zones of metal-poor stars are much shallower than those of Population I stars of comparable mass. Consequently at lower metallicity, convectively driven transport to deep hot layers decreases, and the opportunity for diffusion increases. The convective zone also becomes shallower with increasing effective temperature on the main sequence, so stars nearer the turnoff are similarly expected to have experienced less destruction of $\mathrm{Li}$, but potentially more diffusion.

\section{Observations}

It is against the backdrop of the theoretical framework described above that recent observations have been obtained. The spectra have been pushed to higher quality by two drivers: the desire to measure the intrinsic spread of the stars' $\mathrm{Li}$ abundances about the mean, which provides one constraint on possible lithium depletion mechanisms, and the desire to gain greater certainty over the possible existence of trends of lithium abundance with effective temperature and metallicity, which provide two further constraints on the mechanisms by which photospheric Li abundances have been modified and those mechanisms' effectiveness.

Observations with high $\mathrm{S} / \mathrm{N}(\gtrsim 150$ per pixel) at high resolving power $(R \gtrsim 50000)$ have been obtained by several recent surveys (Ryan, Norris \& Beers 1999; Bonifacio et al. 2003,2005; Asplund 2005; Lambert 2004). All of these exhibit at most a very small spread about the Li plateau at a given effective temperature and metallicity, as low as 0.03 dex, consistent with there being no intrinsic spread once observational and analysis uncertainties are quantified.

There are a few exceptions to this rule in that around $5 \%$ of stars expected to lie on the plateau show no evidence of $\mathrm{Li}$ in their atmospheres at levels well below the plateau. The majority of these exceptions have unusually high rotation velocities for halo stars and are proposed to have unique histories associated with mass transfer events that make them of little use in the quest for the primordial Li abundance (Ryan et al. 2002, Ryan \& Elliott 2005). The extremely metal-poor star HE 1327-2326 is another Li-poor turnoff star, with only a low upper-limit on its $\mathrm{Li}$ abundance. The very non-solar abundance ratios of other elements in this object, however, provide a warning that it might be unreasonable to expect this star to have preserved its Li, even though the precise reason for the star's unusual abundances are still the subject of uncertainty (Frebel et al. 2005).

The question of whether there is a systematic dependence of derived lithium abundance on effective temperature and metallicity is difficult to answer. Taking the data at face value, the high $\mathrm{S} / \mathrm{N}$ studies cited above seem to show such dependences at 
statistically significant levels. The concern that remains is whether such trends are intrinsic to the stars or are an artifact of the analysis procedures, especially in the determination of stellar effective temperatures which is, in my view, the Achilles' heel of Li analyses. (Lithium abundance calculations on the Spite plateau are insensitive to typical uncertainties in gravity, microturbulence and metallicity.) A range of temperature determination techniques was employed in the studies cited above, including optical band photometry, IR photometry and Balmer profile fitting. The results might therefore seem robust to this choice, but it would be a brave stellar atmosphere analyst who would claim that there could be no residual temperature- or metallicity-dependent systematic errors in the derived temperatures. Indeed, recent revisions of infrared flux-method effective temperatures by Meléndez \& Ramírez (2004) have implied substantially higher effective temperatures by 400 to $500 \mathrm{~K}$ for the most metal-poor stars, and this in turn has resulted not only in the derivation of higher abundances but also in the elimination of the metallicity and effective-temperature trends. The large revisions of effective temperature for the most metal-poor stars are a challenge in view of the findings by Arnone et al. (2005) that effective temperatures derived from nulling the dependence of iron abundance on excitation potential were on average $80 \mathrm{~K}$ lower than those derived photometrically, and by Asplund et al. (Lambert 2004) that effective temperatures they derived from $\mathrm{H} \alpha$ profile fits are 50 to $100 \mathrm{~K}$ cooler than photometric ones.

\section{Interpretation of the abundances}

Once the Spite plateau Li abundances are derived, they must be interpreted correctly if one is to infer the primordial value. Although most analyses are conducted using conventional 1D stellar atmosphere analyses and assuming LTE, the change implied by switching to 3D and applying NLTE corrections seems to be very minor (Asplund, Carlsson \& Botnen 2003). Other effects to be considered include possible depletion and diffusion processes, and the possibility of Galactic production of $\mathrm{Li}$ along with the iron and other elements in the gas from which these Population II stars formed. The analysis and error budget presented by Ryan et al. (2000) proposed a primordial abundance $A_{\mathrm{p}}(\mathrm{Li}) \equiv \log _{10}(n(\mathrm{Li}) / n(\mathrm{H}))+12.00=2.09_{-0.13}^{+0.19}$. Broadly similar results were obtained by Bonifacio et al. (2003, 2005) and Asplund (2005) (see Lambert 2004), in the range 1.94 to 2.20, though Meléndez \& Ramírez (2004) find a higher Spite plateau value, $A(\mathrm{Li})$ $=2.37$, on account of their considerably higher effective temperatures for the most metalpoor stars. The analysis of Ryan et al. (2000) assumes no substantial depletion due to destruction, based on the absence of an intrinsic spread about the plateau and in anticipation of the stars with very low Li abundances being shown to be pathological, as now may be established (Ryan et al. 2002; Ryan \& Elliott 2005). Nevertheless, Pinsonneault et al. (2002) favour an analysis leading to an allowance of up to 0.2 dex for depletion due to mixing induced in the stars by shears that result from the spin-down phase of their evolution.

Although the scale of the Spite plateau Li abundance differs in these studies, none finds compelling evidence of a strong decrease in $A(\mathrm{Li})$ in the hottest stars at the mainsequence turnoff, which is the signature that has been the traditionally expected signature of diffusion (Deliyannis \& Demarque 1991, Salaris \& Weiss 2001). However, Richard, Michaud \& Richer (2005) have raised the possibility that turbulent diffusion may occur, the presence of which would not be revealed by this traditional test. They note that as much as 0.5 dex of depletion could occur without preferentially depleting the turnoff stars, depending on the uncertain value of the turbulent diffusion coefficient. If this mechanism 
is in fact active, then substantially higher $A_{\mathrm{p}}(\mathrm{Li})$ values may have to be associated with the observed values $A(\mathrm{Li})$.

What would be the significance of a higher $A_{\mathrm{p}}(\mathrm{Li})$ value? Estimates of the baryonto-photon ratio $\eta$ coming from another primordial nucleosynthesis product, deuterium (O'Meara et al. 2001), and from the angular power spectrum of cosmic microwave background radiation fluctuations (Spergel et al. 2003) both point to a substantially higher value of $\eta$ than can readily be inferred from the Li data (Cyburt, Fields \& Olive 2001; Coc et al. 2002). This embarrassment would be relieved if higher values of $A_{\mathrm{p}}(\mathrm{Li})$ could be reliably inferred.

\section{Li $6104 \AA$}

Given the high quality of observational data now available, it is my view that it is the systematic uncertainty remaining in the effective temperature calibrations, rather than finite $\mathrm{S} / \mathrm{N}$ in the spectra, that imposes the major limit on progress in deriving accurate Li abundances. The Li $6104 \AA$ line may assist with this. Although the $6104 \AA$ line is much weaker than the $6707 \AA$ line, it is nevertheless measurable in the highest $\mathrm{S} / \mathrm{N}$ spectra that are now being acquired for ${ }^{6} \mathrm{Li}$ analyses, e.g. for HD 140283 (Ford et al. 2002). Due to the difference in lower excitation potential of the two lines, $0.00 \mathrm{eV}$ for $6707 \AA$ and $1.85 \mathrm{eV}$ for $6104 \AA$, an error in effective temperature of $100 \mathrm{~K}$ would induce an abundance difference of order 0.03 dex between the two lines. With sufficiently high quality spectra, it should be possible to use the relative equivalent widths of these lines to constrain the effective temperatures.

\section{Acknowledgements}

It is a pleasure to acknowledge fruitful discussions and collaborative studies on Lirelated problems with W. Aoki, T. C. Beers, C. P. Deliyannis, L. Elliott, B. D. Fields, A. Ford, J. E. Norris, K. A. Olive and M. H. Pinsonneault. I would like to dedicate this review to F. Spite and M. Spite whose discovery and interpretation of Li in metal-poor stars became a defining event in the development of my own research career.

\section{References}

Arnone, E., Ryan, S. G., Argast, D., Norris, J. E. \& Beers, T. C. 2005, A\&A A, 430, 507

Asplund, M. 2005 in: L. Pasquini \& S. Randich (eds), Chemical Abundances and Mixing in Stars in the Milky Way and its Satellites in press

Asplund, M., Carlsson, M. \& Botnen, A. V. 2003, A\& A 399, L31

Boesgaard, A. M. \& Steigman, G. 1985, ARAA 23, 319

Bonifacio, P. et al. 2003, IAU JD 15, 39

Bonifacio, P. et al. 2005, this volume

Coc, A., Vangioni-Flam, E., Cassé, M., \& Rabiet, M. 2002, PhysRevD 65, 043510

Cyburt, R., Fields, B. D. \& Olive, K. A. 2001, NewA 6, 215

Deliyannis, C. P. \& Demarque, P. 1991, ApJ 379, 216

Deliyannis, C. P., Demarque, P., \& Kawaler, S. D. 1990, ApJS 73, 21

Ford, A., Jeffries, R. D., Smalley, B., Ryan, S. G., Aoki, W., Kawanomoto, S., James, D. J., \& Barnes, J. R. AEA 393, 617

Frebel et al. 2005, Nature 434, 871

Lambert, D. L. 2004 in: R. E. Allen, D. V. Nanopoulos, and C. N. Pope (eds) The New Cosmology, AIP Conf. Proc. 743, 206 (astro-ph/0410418)

Meléndez, J. \& Ramírez, I. 2004, ApJ 615, L33

O'Meara, J. M., Tytler, D., Kirkman, D., Suzuki, N., Prochaska, J. X., Lubin, D. \& Wolfe, A. M. 2001, ApJ 552, 718 
Pinsonneault, M. H., Deliyannis, C. P., \& Demarque, P. 1992 ApJS 78, 179

Pinsonneault, M. H., Steigman, G., Walker, T. P., \& Narayanan, V. K. 2002, ApJ 574, 398

Richard, O., Michaud, G., \& Richer, J. 2005, ApJ 619, 538

Ryan, S. G., Beers, T. C., Olive, K. A., Fields, B. D., \& Norris, J. E. 2000, ApJ 530, L57

Ryan, S. G. \& Elliott, L. 2005 in: L. Pasquini \& S. Randich (eds), Chemical Abundances and Mixing in Stars in the Milky Way and its Satellites in press (astro-ph/0410472)

Ryan, S. G., Gregory, S., Kolb, U., Beers, T. C., \& Kajino, T. 2002, ApJ 571, 501

Ryan, S. G., Norris, J. E., \& Beers, T. C. 1999, ApJ 523, 654

Salaris, M. \& Weiss, A. 2001, A\&3A 376, 955

Spergel et al. 2003, ApJS 148, 175

Spite, F. \& Spite, M. 1982, A\&A 115, 357 


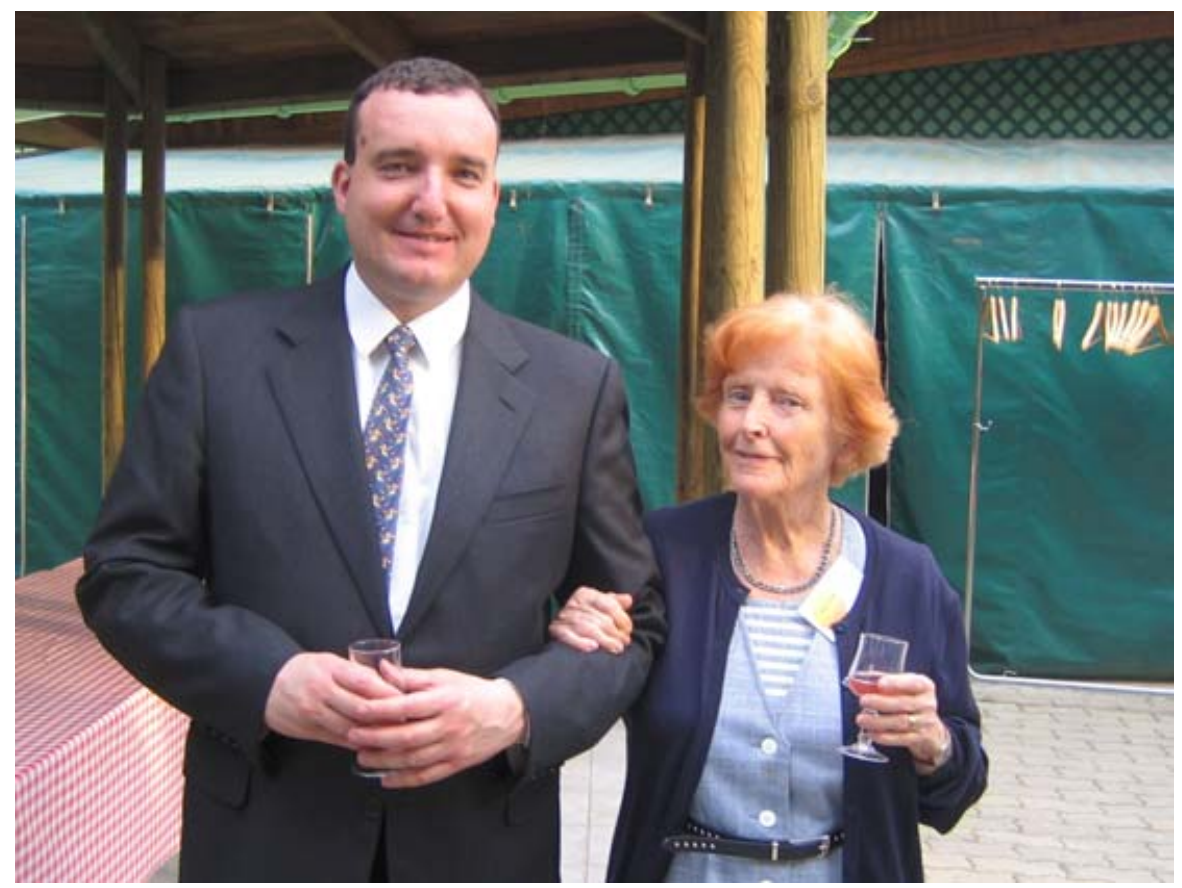

Piercarlo Bonifacio, alongside Giusa Cayrel, at the conference dinner in a Guinguette au bord de la Marne.

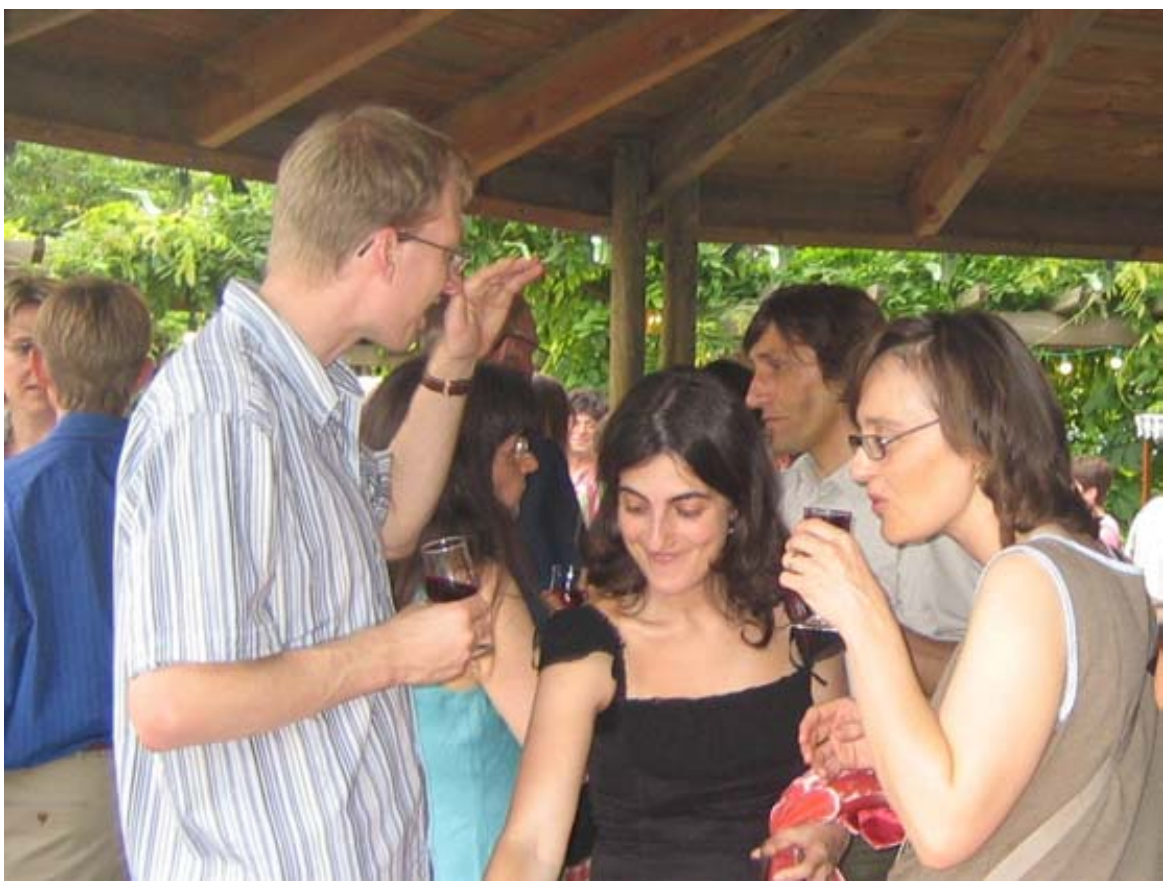

Martin Asplund, Ana Garcìa Pèrez and Francesca Primas, enjoying the aperitif with the other participants, before the conference dinner, held outdoors, on the banks of river Marne, close to Paris. 